Supplement of Biogeosciences, 14, 3253-3274, 2017

https://doi.org/10.5194/bg-14-3253-2017-supplement

(C) Author(s) 2017. This work is distributed under

the Creative Commons Attribution 3.0 License.

(c) (1)

Supplement of

\title{
Modification of the RothC model to simulate soil C mineralization of exogenous organic matter
}

Claudio Mondini et al.

Correspondence to: Claudio Mondini (claudio.mondini@crea.gov.it)

The copyright of individual parts of the supplement might differ from the CC BY 3.0 License. 
Table S1. Incubation conditions, exogenous organic matter (EOM) pool parameters from model optimization, statistical indicators of model goodness of fit and cumulative net respiration for compost amended soils.

\begin{tabular}{|c|c|c|c|c|c|c|c|c|c|c|c|c|c|c|c|c|c|c|}
\hline EOM & Phase & Days & Soil & $\begin{array}{c}\text { Temp } \\
{ }^{\circ} \mathrm{C}\end{array}$ & WHC & $\begin{array}{c}\text { EOM rate } \\
\%\end{array}$ & $\begin{array}{l}\text { C rate } \\
\mu g^{-1}\end{array}$ & $\mathbf{f}_{\text {DEOM }}$ & $\mathbf{f}_{\text {REOM }}$ & $\mathbf{f}_{\text {HEOM }}$ & $\mathbf{K}_{\text {DEOM }}$ & $K_{\text {REOM }}$ & $\begin{array}{c}\text { SSR } \\
\mu \mathrm{Cg}^{-1}\end{array}$ & $\begin{array}{c}\text { RMSE } \\
\%\end{array}$ & $\mathbf{R}$ & $\begin{array}{l}\mathbf{E} \\
\%\end{array}$ & $\begin{array}{c}M \\
\mu g \mathrm{Cg}^{-1}\end{array}$ & $\begin{array}{c}\mathrm{CO}_{2}-\mathrm{C} \\
\%\end{array}$ \\
\hline VSC & $\mathrm{MC}$ & 30 & SM & 20 & 40 & 0.5 & 1725 & 0.02 & 0.39 & 0.59 & 45 & 0.35 & 42 & 1.3 & 0.9994 & 0.33 & 0.11 & 3.0 \\
\hline VSC & MC & 30 & PE & 20 & 40 & 0.5 & 1725 & 0.02 & 0.44 & 0.54 & 58 & 0.25 & 193 & 2.9 & 0.9972 & -0.03 & -0.01 & 2.8 \\
\hline VSC & MC & 32 & $\mathrm{LL}$ & 20 & 40 & 0.5 & 1725 & 0.00 & 0.44 & 0.56 & 150 & 0.15 & 137 & 9.5 & 0.9756 & 0.25 & 0.02 & 0.9 \\
\hline VSC & MC & 29 & $\mathrm{AL}$ & 20 & 40 & 0.5 & 1725 & 0.01 & 0.30 & 0.69 & 224 & 0.18 & 25 & 3.5 & 0.9957 & -0.05 & -0.01 & 1.0 \\
\hline $\mathrm{HWC}$ & $\mathrm{MC}$ & 30 & SM & 20 & 40 & 0.5 & 1720 & 0.02 & 0.37 & 0.61 & 43 & 0.35 & 40 & 1.3 & 0.9995 & 0.31 & 0.11 & 3.0 \\
\hline HWC & $M C$ & 32 & SM & 20 & 40 & 0.5 & 1720 & 0.02 & 0.33 & 0.65 & 43 & 0.15 & 36 & 1.6 & 0.9990 & 0.19 & 0.06 & 2.6 \\
\hline HWC & $M C$ & 32 & SM & 20 & 40 & 0.5 & 1720 & 0.02 & 0.34 & 0.63 & 41 & 0.15 & 87 & 2.6 & 0.9976 & 0.27 & 0.09 & 2.5 \\
\hline HWC & MC & 35 & SM & 30 & 40 & 0.5 & 1720 & 0.02 & 0.30 & 0.68 & 74 & 0.15 & 179 & 3.2 & 0.9930 & 0.15 & 0.06 & 2.6 \\
\hline HWC & MC & 35 & SM & 30 & 40 & 0.5 & 1720 & 0.01 & 0.35 & 0.64 & 93 & 0.15 & 334 & 5.6 & 0.9825 & 0.19 & 0.06 & 2.1 \\
\hline HWC & $M C$ & 36 & SM & 10 & 40 & 0.5 & 1720 & 0.02 & 0.34 & 0.64 & 44 & 0.15 & 20 & 2.1 & 0.9995 & 1.53 & 0.28 & 1.6 \\
\hline HWC & $M C$ & 36 & SM & 10 & 40 & 0.5 & 1720 & 0.02 & 0.37 & 0.60 & 35 & 0.15 & 10 & 1.4 & 0.9996 & 0.22 & 0.04 & 1.8 \\
\hline HWC & MC & 28 & SM & 20 & 20 & 0.5 & 1720 & 0.01 & 0.31 & 0.68 & 118 & 0.29 & 65 & 3.8 & 0.9932 & -0.41 & -0.09 & 1.6 \\
\hline HWC & MC & 28 & SM & 20 & 30 & 0.5 & 1720 & 0.01 & 0.53 & 0.46 & 127 & 0.19 & 35 & 3.0 & 0.9958 & 0.74 & 0.14 & 1.5 \\
\hline HWC & $M C$ & 34 & SM & 20 & 40 & 0.1 & 344 & 0.02 & 0.28 & 0.70 & 54 & 0.86 & 6 & 2.3 & 0.9987 & 0.39 & 0.04 & 4.3 \\
\hline HWC & $M C$ & 34 & SM & 20 & 40 & 0.25 & 860 & 0.02 & 0.28 & 0.70 & 54 & 0.39 & 17 & 2.4 & 0.9984 & 0.42 & 0.07 & 2.6 \\
\hline HWC & MC & 30 & $\mathrm{AL}$ & 20 & 40 & 0.5 & 1720 & 0.01 & 0.26 & 0.72 & 72 & 0.46 & 46 & 2.5 & 0.9981 & 0.64 & 0.14 & 2.0 \\
\hline GWB & $M C$ & 13 & $P E$ & 20 & 40 & 0.75 & 2115 & 0.01 & 0.52 & 0.47 & 200 & 0.42 & 21 & 2.7 & 0.9978 & 0.72 & 0.13 & 1.3 \\
\hline GWB & $M C$ & 13 & GO & 20 & 40 & 0.75 & 2115 & 0.01 & 0.40 & 0.59 & 200 & 0.15 & 10 & 1.9 & 0.9983 & 0.28 & 0.05 & 1.0 \\
\hline $\mathrm{CMC}$ & II & 37 & JU & 20 & 40 & 0.5 & 2135 & 0.04 & 0.89 & 0.07 & 38 & 0.31 & 115 & 0.8 & 0.9998 & -0.02 & -0.02 & 6.1 \\
\hline $\mathrm{CMC}$ & III & 37 & JU & 20 & 40 & 0.5 & 2063 & 0.05 & 0.63 & 0.32 & 28 & 0.33 & 173 & 1.0 & 0.9997 & -0.10 & -0.08 & 6.0 \\
\hline $\mathrm{CMC}$ & $M C$ & 37 & JU & 20 & 40 & 0.5 & 1993 & 0.002 & 0.31 & 0.69 & 105 & 0.36 & 48 & 3.1 & 0.9983 & 0.41 & 0.06 & 1.4 \\
\hline $\mathrm{CBC}$ & II & 37 & JU & 20 & 40 & 0.5 & 2174 & 0.07 & 0.74 & 0.19 & 22 & 0.23 & 94 & 0.7 & 0.9999 & -0.03 & -0.03 & 6.6 \\
\hline $\mathrm{CBC}$ & III & 37 & JU & 20 & 40 & 0.5 & 2124 & 0.07 & 0.59 & 0.34 & 18 & 0.16 & 651 & 2.1 & 0.9989 & -0.35 & -0.27 & 5.6 \\
\hline $\mathrm{CBC}$ & IV & 37 & JU & 20 & 40 & 0.5 & 2117 & 0.05 & 0.57 & 0.38 & 16 & 0.23 & 3981 & 6.5 & 0.9952 & -0.98 & -0.62 & 4.9 \\
\hline $\mathrm{CBC}$ & $M C$ & 37 & JU & 20 & 40 & 0.5 & 2031 & 0.01 & 0.35 & 0.64 & 99 & 0.38 & 113 & 3.1 & 0.9979 & -0.68 & -0.15 & 1.9 \\
\hline $\mathrm{CMC}$ & II & 37 & JU & 30 & 40 & 0.5 & 2135 & 0.06 & 0.84 & 0.10 & 45 & 0.37 & 494 & 1.0 & 0.9996 & 0.02 & 0.03 & 9.6 \\
\hline $\mathrm{CMC}$ & III & 37 & JU & 30 & 40 & 0.5 & 2063 & 0.06 & 0.62 & 0.32 & 30 & 0.38 & 590 & 1.3 & 0.9994 & 0.00 & 0.00 & 8.5 \\
\hline CMC & $M C$ & 37 & JU & 30 & 40 & 0.5 & 1993 & 0.01 & 0.21 & 0.78 & 62 & 0.57 & 181 & 2.9 & 0.9982 & 0.21 & 0.07 & 2.7 \\
\hline $\mathrm{CBC}$ & II & 37 & JU & 30 & 40 & 0.5 & 2174 & 0.09 & 0.71 & 0.20 & 21 & 0.33 & 1897 & 1.7 & 0.9990 & 0.18 & 0.30 & 11.1 \\
\hline $\mathrm{CBC}$ & III & 37 & JU & 30 & 40 & 0.5 & 2124 & 0.07 & 0.65 & 0.28 & 21 & 0.37 & 953 & 1.6 & 0.9997 & -0.02 & -0.03 & 9.3 \\
\hline mean & & & & & & & & 0.03 & 0.44 & 0.53 & 79 & 0.30 & 403 & 2.7 & 0.9968 & 0.11 & 0.01 & 3.7 \\
\hline minimum & & & & & & & & 0.00 & 0.21 & 0.07 & 16 & 0.15 & 6 & 0.7 & 0.9756 & -1.46 & -0.62 & 0.9 \\
\hline maximum & & & & & & & & 0.09 & 0.89 & 0.78 & 224 & 0.86 & 3981 & 9.5 & 0.9999 & 1.53 & 0.30 & 11.1 \\
\hline
\end{tabular}

861 EOM: exogenous organic matter; WHC: water holding capacity; DEOM: decomposable EOM; REOM: resistant

862 EOM; HEOM: humified EOM; f: partitioning coefficient; K: decomposition constant rate $\left(\mathrm{y}^{-1}\right)$; SSR: sum of

863 squared residuals; RMSE: root mean square error; R: coefficient of correlation; E: relative error; M: mean

864 difference; $\mathrm{CO}_{2}-\mathrm{C}$ : cumulative net $\mathrm{CO}_{2}-\mathrm{C}$ emission in amended soil; $\mathrm{MC}$ : mature compost; roman numerals refer

865 to phases of the composting process. For EOM code refer to Table 3, for soil code refer to Table 2. 
Table S2. Incubation conditions, exogenous organic matter (EOM) pool parameters from model optimization, statistical indicators of model goodness of fit and cumulative net respiration for bioenergy by-products.

\begin{tabular}{|c|c|c|c|c|c|c|c|c|c|c|c|c|c|c|c|c|}
\hline EOM & Days & Soil & $\begin{array}{c}\text { Temp } \\
{ }^{\circ} \mathrm{C}\end{array}$ & WHC & $\begin{array}{c}\text { EOM rate } \\
\%\end{array}$ & $\begin{array}{l}\text { C rate } \\
\mu g^{-1}\end{array}$ & $f_{D E O M}$ & $\mathbf{f}_{\text {REOM }}$ & $K_{\text {DEOM }}$ & $\mathrm{K}_{\text {REOM }}$ & $\begin{array}{c}\text { SSR } \\
\mu \mathrm{C}^{-1}\end{array}$ & $\begin{array}{c}\text { RMSE } \\
\%\end{array}$ & $\mathbf{R}$ & $\begin{array}{c}\mathbf{E} \\
\%\end{array}$ & $\begin{array}{c}M \\
\mu g \mathrm{Cg}^{-1}\end{array}$ & $\begin{array}{c}\mathrm{CO}_{2}-\mathrm{C} \\
\%\end{array}$ \\
\hline$B R$ & 30 & SM & 20 & 40 & 0.5 & 2425 & 0.12 & 0.88 & 147 & 0.68 & 30347 & 4.3 & 0.990 & -1.47 & -4.27 & 15.1 \\
\hline$B R$ & 30 & PE & 20 & 40 & 0.5 & 2425 & 0.15 & 0.85 & 145 & 0.37 & 8416 & 2.2 & 0.995 & 0.17 & 0.50 & 14.3 \\
\hline$B R$ & 34 & SM & 20 & 40 & 0.1 & 485 & 0.11 & 0.89 & 124 & 0.52 & 233 & 2.6 & 0.995 & 0.29 & 0.16 & 13.7 \\
\hline$B R$ & 32 & $\mathrm{LL}$ & 20 & 40 & 0.5 & 2425 & 0.11 & 0.89 & 113 & 0.29 & 1835 & 1.3 & 0.998 & -0.01 & -0.02 & 10.9 \\
\hline $\mathrm{BR}$ & 29 & $A L$ & 20 & 40 & 0.5 & 2425 & 0.20 & 0.80 & 81 & 0.15 & 152617 & 9.5 & 0.985 & -0.42 & -1.39 & 16.8 \\
\hline RSM & 36 & SM & 10 & 40 & 0.5 & 2295 & 0.04 & 0.96 & 186 & 1.36 & 6075 & 5.3 & 0.991 & -0.26 & -0.33 & 8.5 \\
\hline RSM & 30 & $\mathrm{SM}$ & 20 & 40 & 0.5 & 2295 & 0.11 & 0.89 & 44 & 0.32 & 3128 & 2.0 & 0.998 & -0.04 & -0.09 & 11.8 \\
\hline RSM & 32 & $\mathrm{SM}$ & 20 & 40 & 0.5 & 2295 & 0.15 & 0.85 & 65 & 0.33 & 3102 & 1.9 & 0.998 & 0.07 & 0.20 & 14.7 \\
\hline RSM & 32 & SM & 20 & 40 & 0.5 & 2295 & 0.16 & 0.84 & 71 & 0.32 & 2583 & 1.5 & 0.999 & 0.14 & 0.42 & 16.0 \\
\hline RSM & 35 & SM & 30 & 40 & 0.5 & 2295 & 0.13 & 0.87 & 108 & 0.18 & 3467 & 1.9 & 0.996 & 0.00 & -0.01 & 13.5 \\
\hline RSM & 35 & SM & 30 & 40 & 0.5 & 2295 & 0.14 & 0.86 & 125 & 0.17 & 5010 & 2.2 & 0.994 & 0.21 & 0.61 & 14.0 \\
\hline RSM & 34 & SM & 20 & 40 & 0.1 & 459 & 0.13 & 0.87 & 75 & 0.41 & 78 & 1.6 & 0.999 & -0.08 & -0.04 & 14.1 \\
\hline RSM & 32 & $\amalg$ & 20 & 40 & 0.5 & 2295 & 0.07 & 0.93 & 49 & 0.15 & 810 & 1.6 & 0.999 & -0.16 & -0.19 & 6.9 \\
\hline RSM & 29 & $\mathrm{AL}$ & 20 & 40 & 0.5 & 2295 & 0.11 & 0.89 & 77 & 0.15 & 92204 & 11.5 & 0.989 & -6.44 & -13.70 & 9.6 \\
\hline mean & & & & & & & 0.12 & 0.88 & 98 & 0.42 & 16043 & 3.1 & 0.996 & -0.42 & -0.95 & 12.9 \\
\hline minimum & & & & & & & 0.04 & 0.80 & 44 & 0.15 & 78 & 1.1 & 0.985 & -6.44 & -13.70 & 6.9 \\
\hline maximum & & & & & & & 0.20 & 0.96 & 186 & 1.36 & 152617 & 11.5 & 0.999 & 0.30 & 0.61 & 16.8 \\
\hline
\end{tabular}

EOM: exogenous organic matter; WHC: water holding capacity; DEOM: decomposable EOM; REOM: resistant EOM; HEOM: humified EOM; f: partitioning factor; K:

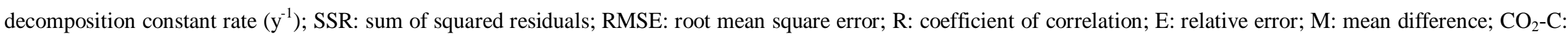
cumulative net $\mathrm{CO}_{2}-\mathrm{C}$ emission in amended soil.

For EOM code refer to Table 3, for soil code refer to Table 2. 
873 Table S3. Incubation conditions, EOM pool parameters from model optimization, statistical indicators of model goodness of fit and cumulative net respiration for anaerobic digestates.

\begin{tabular}{|c|c|c|c|c|c|c|c|c|c|c|c|c|c|c|c|c|c|}
\hline EOM & Days & Soil & $\begin{array}{c}\text { Temp } \\
{ }^{\circ} \mathrm{C}\end{array}$ & WHC & $\begin{array}{c}\text { EOM rate } \\
\%\end{array}$ & $\begin{array}{l}\text { C rate } \\
\mu g^{-1}\end{array}$ & $f_{D E O M}$ & $f_{\text {REOM }}$ & $f_{\text {HEOM }}$ & $K_{D E O M}$ & $\mathrm{~K}_{\text {REOM }}$ & $\begin{array}{c}\text { SSR } \\
\mu g \mathrm{Cg}^{-1}\end{array}$ & $\begin{array}{c}\text { RMSE } \\
\%\end{array}$ & $\mathbf{R}$ & $\begin{array}{l}\mathbf{E} \\
\%\end{array}$ & $\begin{array}{c}M \\
\mu \mathrm{gC} g^{-1}\end{array}$ & $\begin{array}{c}\mathrm{CO}_{2}-\mathrm{C} \\
\%\end{array}$ \\
\hline PS & 36 & SM & 10 & 40 & 0.50 & 1895 & 0.02 & 0.84 & 0.15 & 40 & 0.86 & 621 & 5.0 & 0.9961 & -0.58 & -0.2 & 3 \\
\hline PS & 36 & SM & 10 & 40 & 0.50 & 1895 & 0.00 & 0.70 & 0.30 & 187 & 1.52 & 727 & 5.4 & 9953 & 0.14 & 0.1 & \\
\hline PS & 30 & SM & 20 & 40 & 0.50 & 1895 & 0.08 & 0.62 & 0.30 & 26 & 0.15 & 345 & 1.5 & 9995 & -0.25 & -0.2 & \\
\hline PS & 32 & SM & 20 & 40 & 0.50 & 1895 & 0.06 & 0.64 & 0.30 & 38 & 0.21 & 122 & 1.1 & 9996 & 0.11 & 0.1 & 6. \\
\hline PS & 32 & SM & 20 & 40 & 0.50 & 1895 & 0.07 & 0.63 & 0.30 & 52 & 0.21 & 305 & 1.6 & 9989 & -0.40 & -0.4 & 6. \\
\hline PS & 35 & SM & 30 & 40 & 0.50 & 1895 & 0.05 & 0.65 & 0.30 & 73 & 0.15 & 1328 & 3.6 & 9892 & -0.35 & -0.3 & J. \\
\hline PS & 35 & SM & 30 & 40 & 0.50 & 1895 & 0.04 & 0.66 & 0.30 & 84 & 0.15 & 701 & 2.8 & 9951 & 1.47 & 1.2 & \\
\hline PS & 28 & SM & 20 & 20 & & 1895 & & 0.81 & & 72 & 0.32 & 235 & 2.2 & & -0.32 & -0.2 & \\
\hline PS & 28 & SM & 20 & 30 & & 1895 & & & 0. & 69 & & 126 & 1.6 & & -0.19 & -0.1 & \\
\hline PS & 30 & $P E$ & 20 & 40 & & 1895 & & 0.90 & 0.06 & 70 & 0.28 & 98 & 1.0 & & 0.05 & 0.0 & \\
\hline PS & 34 & SM & 20 & 40 & 0.10 & 379 & 0.05 & 0.75 & 0.20 & 55 & 0.43 & 13 & 1.7 & 9990 & 0.25 & 0.0 & 7.2 \\
\hline PS & 34 & SM & 20 & 40 & 0.25 & 948 & 0.05 & 0.65 & 0.30 & 57 & 0.44 & 45 & 1.4 & 9993 & 0.16 & 0.1 & 6.7 \\
\hline PS & 32 & L & 20 & 40 & & 1895 & & 0.67 & 0.30 & 41 & 0.15 & 111 & 1.5 & 9991 & -0.02 & 0.0 & 3.4 \\
\hline PS & 29 & $\mathrm{AL}$ & 20 & 40 & 0.50 & 1895 & 0.05 & 0.73 & 0.22 & 47 & 0.16 & 303 & 2.2 & 0.9987 & -0.12 & -0.1 & 4.9 \\
\hline OW1 & 21 & SM & 20 & 40 & & 325 & 0.00 & 0.70 & 0.30 & 270 & 0.15 & 7 & 18.8 & 0.9446 & 2.67 & 0.0 & 0.8 \\
\hline OW1 & 26 & SM & 20 & 40 & 1.5 & 64 & 1 & 0.83 & 16 & 64 & 17 & 5 & 3.0 & 9989 & 0.58 & 0.0 & 2. \\
\hline OW1 & 10 & $\mathrm{CO}$ & 20 & 40 & & 649 & 0.01 & 0.78 & 0.21 & 260 & 0.15 & 9 & 4.81 & 0.9919 & 3.39 & 0.1 & \\
\hline OW2 & 13 & SM & 20 & 40 & 0.75 & 347 & 0.01 & 0.83 & 0.16 & 151 & 0.20 & 2 & 5.1 & 0.9943 & 0.95 & 0.0 & \\
\hline OW2 & 23 & SM & 20 & 40 & 1.50 & 693 & 0.02 & 0.79 & 0.19 & 151 & 0.44 & 1 & 1.6 & 0.9995 & -0.20 & 0.0 & 3. \\
\hline OW2 & 7 & $\mathrm{CO}$ & 20 & 40 & 1.50 & 693 & 0.01 & 0.69 & 0.30 & 267 & 0.17 & 6 & 3.9 & 0.9967 & 0.93 & 0.1 & 1. \\
\hline OW3 & 26 & SM & 20 & 40 & 0.7 & 362 & 0.0 & 0.70 & 0.30 & 280 & 0.15 & 51 & 37.2 & 0.7938 & 1.08 & 0.0 & 0.9 \\
\hline OW3 & 22 & SM & 20 & 40 & 1.50 & 723 & 0.6 & 0.69 & 0.30 & 72 & 0.15 & 39 & 9.2 & 0.9878 & -1.60 & -0.1 & 1.8 \\
\hline OW3 & 7 & CO & 20 & 40 & 1.50 & 723 & 0.01 & 0.69 & 0.30 & 279 & 0.15 & 9 & 5.5 & 0.9903 & 1.18 & 0.1 & 1. \\
\hline OW4 & 26 & SM & 20 & 40 & 0.75 & 404 & 0.01 & 0.69 & 0.30 & 280 & 0.15 & 31 & 13.8 & 0.9645 & 1.75 & 0.1 & 1. \\
\hline OW4 & 22 & SM & 20 & 40 & 1.50 & 807 & 0.0 & 0.84 & 0.15 & 230 & 0.39 & 48 & 7.7 & 0.9892 & 0.24 & 0.0 & \\
\hline OW4 & 7 & $\mathrm{CO}$ & 20 & 40 & & 807 & & 0.69 & 0.30 & 330 & 0.15 & 9 & 3.6 & 0.9943 & 0.55 & 0.1 & \\
\hline OW4 & 13 & $\mathrm{PE}$ & 20 & 40 & 1.50 & 807 & 0.02 & 0.68 & 0.30 & 229 & 0.19 & 116 & 7.9 & 0.9793 & 3.16 & 0.5 & 2. \\
\hline & & & & & & & 0.03 & 0.72 & 0.25 & 140 & 0.30 & 201 & 5.7 & 0.9849 & 0.54 & 0.0 & 3. \\
\hline minimu & & & & & & & 0.00 & 0.62 & 0.06 & 26 & 0.15 & 1 & 1.0 & 0.7938 & -1.60 & -0.4 & 0.8 \\
\hline maximum & & & & & & & 0.08 & 0.90 & 0.30 & 330 & 1.52 & 1328 & 37 & 0.9996 & 3.39 & 1.2 & 7.2 \\
\hline
\end{tabular}

876 EOM: exogenous organic matter; WHC: water holding capacity; DEOM: decomposable EOM; REOM: resistant 877 EOM; HEOM: humified EOM; f: partitioning coefficient; K: decomposition constant rate $\left(\mathrm{y}^{-1}\right)$; SSR: sum of 878 squared residuals; RMSE: root mean square error; R: coefficient of correlation; E: relative error; M: mean 879 difference; $\mathrm{CO}_{2}-\mathrm{C}$ : cumulative net $\mathrm{CO}_{2}-\mathrm{C}$ emission in amended soil.

880 For EOM code refer to Table 3, for soil code refer to Table 2. 
Table S4a. Incubation conditions, exogenous organic matter (EOM) pool parameters from model optimization, statistical indicators of model goodness of fit and cumulative net respiration for meat and bone meals.

\begin{tabular}{|c|c|c|c|c|c|c|c|c|c|c|c|c|c|c|c|c|}
\hline EOM & Days & Soil & $\begin{array}{c}\text { Temp } \\
{ }^{\circ} \mathrm{C}\end{array}$ & WHC & $\begin{array}{c}\text { EOM rate } \\
\mu \mathrm{gCg} g^{-1}\end{array}$ & $\begin{array}{l}\text { EOM rate } \\
\mathrm{kg} \mathrm{N} \mathrm{ha}^{-1}\end{array}$ & $f_{\text {DEOM }}$ & $f_{\text {REOM }}$ & $K_{\text {DEOM }}$ & $K_{\text {REOM }}$ & $\begin{array}{c}\text { SSR } \\
\mu g \mathrm{Cg}^{-1}\end{array}$ & $\begin{array}{c}\text { RMSE } \\
\%\end{array}$ & $\mathbf{R}$ & $\begin{array}{l}\mathbf{E} \\
\%\end{array}$ & $\begin{array}{c}M \\
\mu g \mathrm{Cg}^{-1}\end{array}$ & $\begin{array}{c}\mathrm{CO}_{2}-\mathrm{C} \\
\%\end{array}$ \\
\hline $\begin{array}{l}\text { BV1 } \\
\text { BV1 }\end{array}$ & $\begin{array}{l}14 \\
14\end{array}$ & $\begin{array}{l}\mathrm{PE} \\
\mathrm{PE}\end{array}$ & $\begin{array}{l}20 \\
25\end{array}$ & $\begin{array}{l}40 \\
40\end{array}$ & $\begin{array}{l}961 \\
961\end{array}$ & $\begin{array}{l}800 \\
800\end{array}$ & $\begin{array}{l}0.15 \\
0.21\end{array}$ & $\begin{array}{l}0.85 \\
0.79\end{array}$ & $\begin{array}{l}89 \\
90\end{array}$ & $\begin{array}{l}1.15 \\
1.41\end{array}$ & $\begin{array}{r}1449 \\
507\end{array}$ & $\begin{array}{l}3.8 \\
1.5\end{array}$ & $\begin{array}{l}0.997 \\
0.999\end{array}$ & $\begin{array}{l}-1.05 \\
-0.15\end{array}$ & $\begin{array}{l}-1.12 \\
-0.23\end{array}$ & $\begin{array}{l}15.5 \\
22.1\end{array}$ \\
\hline BV1 & 12 & $\mathrm{BU}$ & 20 & 40 & 240 & 200 & 0.19 & 0.81 & 51 & 0.20 & 359 & 11.2 & 0.992 & -6.37 & -1.22 & 12.8 \\
\hline BV1 & 12 & BU & 20 & 40 & 480 & 400 & 0.17 & 0.83 & 64 & 0.18 & 1619 & 11.8 & 0.991 & -6.69 & -2.58 & 12.3 \\
\hline BV1 & 12 & LO & 20 & 40 & 240 & 200 & 0.31 & 0.69 & 83 & 0.22 & 359 & 5.1 & 0.997 & -1.83 & -0.77 & 24.3 \\
\hline BV1 & 12 & LO & 20 & 40 & 480 & 400 & 0.33 & 0.67 & 81 & 0.15 & 1377 & 4.7 & 0.996 & -1.38 & -1.23 & 25.5 \\
\hline BV1 & 13 & GO & 20 & 40 & 120 & 100 & 0.26 & 0.74 & 64 & 0.15 & 34 & 3.9 & 0.997 & 0.38 & 0.07 & 20.3 \\
\hline BV1 & 13 & SM & 20 & 40 & 120 & 100 & 0.27 & 0.73 & 71 & 0.43 & 102 & 6.0 & 0.996 & -2.21 & -0.43 & 23.1 \\
\hline BV1 & 13 & GO & 20 & 40 & 240 & 200 & 0.23 & 0.77 & 74 & 0.15 & 121 & 4.1 & 0.996 & -0.67 & -0.21 & 17.9 \\
\hline BV1 & 13 & SM & 20 & 40 & 240 & 200 & 0.24 & 0.76 & 65 & 0.35 & 413 & 7.3 & 0.996 & -3.59 & -1.15 & 19.8 \\
\hline BV1 & 13 & GO & 20 & 40 & 480 & 400 & 0.24 & 0.76 & 74 & 0.15 & 588 & 4.3 & 0.996 & -0.72 & -0.46 & 18.7 \\
\hline BV1 & 13 & SM & 20 & 40 & 481 & 400 & 0.24 & 0.76 & 65 & 0.33 & 1796 & 7.4 & 0.996 & -3.75 & -2.46 & 20.2 \\
\hline BV1 & 14 & SM & 20 & 40 & 961 & 800 & 0.16 & 0.84 & 66 & 0.23 & 6376 & 9.9 & 0.993 & -6.28 & -5.52 & 13.5 \\
\hline BV1 & 14 & PE & 25 & 40 & 240 & 200 & 0.18 & 0.82 & 79 & 0.16 & 36 & 2.3 & 0.998 & -0.18 & -0.05 & 15.3 \\
\hline BV1 & 14 & SM & 25 & 40 & 240 & 200 & 0.15 & 0.85 & 83 & 0.15 & 121 & 4.7 & 0.997 & -1.90 & -0.48 & 13.1 \\
\hline BV1 & 14 & PE & 25 & 40 & 481 & 400 & 0.18 & 0.82 & 77 & 0.49 & 219 & 2.8 & 0.999 & -0.91 & -0.53 & 15.9 \\
\hline BV1 & 14 & SM & 25 & 40 & 481 & 400 & 0.16 & 0.84 & 75 & 0.20 & 706 & 5.5 & 0.996 & -2.50 & -1.31 & 14.4 \\
\hline BV1 & 14 & SM & 25 & 40 & 961 & 800 & 0.22 & 0.78 & 55 & 0.15 & 2654 & 4.2 & 0.995 & -0.15 & -0.20 & 18.3 \\
\hline BV1 & 13 & $\mathrm{PE}$ & 20 & 40 & 114 & 100 & 0.14 & 0.86 & 85 & 1.45 & 6 & 2.2 & 0.999 & -0.22 & -0.03 & 15.4 \\
\hline BV1 & 13 & $\mathrm{PE}$ & 20 & 40 & 228 & 200 & 0.13 & 0.87 & 109 & 1.03 & 13 & 1.7 & 0.999 & -0.005 & -0.001 & 13.4 \\
\hline BV1 & 14 & SM & 20 & 40 & 114 & 100 & 0.15 & 0.85 & 82 & 0.45 & 13 & 3.2 & 0.998 & -0.77 & -0.09 & 14.3 \\
\hline BV1 & 14 & SM & 20 & 40 & 228 & 200 & 0.14 & 0.86 & 85 & 0.32 & 54 & 3.4 & 0.997 & -0.72 & -0.16 & 13.1 \\
\hline BV1 & 14 & SM & 20 & 40 & 455 & 400 & 0.14 & 0.86 & 100 & 0.69 & 440 & 4.4 & 0.997 & -1.70 & -0.83 & 14.5 \\
\hline BV1 & 14 & SM & 20 & 40 & 683 & 600 & 0.15 & 0.85 & 92 & 0.62 & 739 & 3.9 & 0.998 & -1.56 & -1.13 & 14.5 \\
\hline BV1 & 13 & PE & 20 & 40 & 455 & 400 & 0.17 & 0.83 & 88 & 0.89 & 100 & 2.1 & 0.999 & -0.16 & -0.08 & 15.9 \\
\hline BV1 & 13 & $\mathrm{PE}$ & 20 & 40 & 683 & 600 & 0.15 & 0.85 & 90 & 0.53 & 224 & 2.4 & 0.998 & -0.26 & -0.17 & 13.7 \\
\hline
\end{tabular}

886 EOM: exogenous organic matter; WHC: water holding capacity; DEOM: decomposable EOM; REOM: resistant

887 EOM; HEOM: humified EOM; f: partitioning coefficient; K: decomposition constant rate $\left(\mathrm{y}^{-1}\right)$; SSR: sum of

888 squared residuals; RMSE: root mean square error; R: coefficient of correlation; E: relative error; M: mean 889 difference; $\mathrm{CO}_{2}-\mathrm{C}$ : cumulative net $\mathrm{CO}_{2}-\mathrm{C}$ emission in amended soil.

890 For EOM code refer to Table 3, for soil code refer to Table 2. 
Table S4b. Incubation conditions, exogenous organic matter (EOM) pool parameters from model optimization, statistical indicators of model goodness of fit and cumulative net respiration for meat and bone meals.

\begin{tabular}{|c|c|c|c|c|c|c|c|c|c|c|c|c|c|c|c|c|}
\hline EOM & Days & Soil & $\begin{array}{l}\text { Temp } \\
{ }^{\circ} \mathrm{C}\end{array}$ & WHC & $\begin{array}{c}\text { EOM rate } \\
\mu \mathrm{gCg}^{-1}\end{array}$ & $\begin{array}{l}\text { EOM rate } \\
\mathrm{kg} \mathrm{N} \mathrm{ha}^{-1}\end{array}$ & $f_{D E O M}$ & $f_{\text {REOM }}$ & $\mathrm{K}_{\mathrm{DEOM}}$ & $K_{\text {REOM }}$ & $\begin{array}{c}\text { SSR } \\
\mu \mathrm{gCg} \mathrm{g}^{-1}\end{array}$ & $\begin{array}{c}\text { RMSE } \\
\%\end{array}$ & $\mathbf{R}$ & $\begin{array}{l}\mathbf{E} \\
\%\end{array}$ & $\begin{array}{c}M \\
\mu \mathrm{gCg} g^{-1}\end{array}$ & $\begin{array}{c}\mathrm{CO}_{2}-\mathrm{C} \\
\%\end{array}$ \\
\hline SB & 9 & $\mathrm{BU}$ & 20 & 40 & 211 & 200 & 0.63 & 0.37 & 13 & 0.15 & 150 & 8.9 & 0.994 & -4.74 & -0.85 & 16.1 \\
\hline SB & 9 & LO & 20 & 40 & 211 & 200 & 0.60 & 0.40 & 52 & 0.32 & 88 & 2.5 & 0.999 & -0.66 & -0.33 & 38.6 \\
\hline SB & 9 & SM & 20 & 40 & 211 & 200 & 0.45 & 0.55 & 40 & 0.26 & 189 & 5.3 & 0.998 & -2.66 & -0.90 & 27.2 \\
\hline SB & 16 & $\mathrm{RI}$ & 15 & 40 & 211 & 200 & 0.45 & 0.55 & 42 & 0.41 & 283 & 4.4 & 0.997 & -0.82 & -0.31 & 28.7 \\
\hline SB & 13 & $\mathrm{RI}$ & 20 & 40 & 211 & 200 & 0.37 & 0.63 & 42 & 0.20 & 409 & 7.7 & 0.997 & -3.60 & -1.22 & 25.1 \\
\hline SB & 16 & JU & 15 & 40 & 211 & 200 & 0.17 & 0.83 & 56 & 0.15 & 540 & 16.1 & 0.991 & -11.18 & -1.63 & 11.6 \\
\hline SB & 16 & PE & 15 & 40 & 211 & 200 & 0.15 & 0.85 & 75 & 0.23 & 127 & 6.8 & 0.995 & -2.65 & -0.44 & 11.7 \\
\hline SB & 18 & SM & 15 & 40 & 211 & 200 & 0.15 & 0.85 & 83 & 0.26 & 633 & 13.4 & 0.989 & -8.08 & -1.46 & 12.5 \\
\hline SB & 9 & GO & 20 & 40 & 211 & 200 & 0.36 & 0.64 & 48 & 0.15 & 214 & 6.3 & 0.994 & -0.54 & -0.16 & 22.6 \\
\hline SB & 13 & JU & 20 & 40 & 211 & 200 & 0.20 & 0.80 & 58 & 0.18 & 169 & 8.1 & 0.996 & -3.98 & -0.82 & 14.5 \\
\hline SB & 13 & PE & 20 & 40 & 211 & 200 & 0.24 & 0.76 & 50 & 0.25 & 52 & 3.8 & 0.999 & -1.72 & -0.42 & 17.4 \\
\hline SB & 27 & JU & 20 & 40 & 2460 & 2220 & 0.19 & 0.81 & 58 & 0.90 & 6498 & 1.8 & 0.999 & -0.15 & -0.52 & 20.0 \\
\hline SB & 27 & LO & 20 & 40 & 2460 & 2220 & 0.30 & 0.70 & 77 & 0.30 & 41474 & 2.9 & 0.996 & -0.35 & -1.84 & 25.9 \\
\hline SB & 26 & SM & 20 & 40 & 2460 & 2220 & 0.21 & 0.79 & 27 & 0.36 & 16202 & 3.9 & 0.997 & 0.75 & 2.11 & 18.1 \\
\hline SB & 26 & SM & 20 & 40 & 4920 & 4440 & 0.07 & 0.93 & 101 & 0.92 & 27626 & 3.4 & 0.996 & 0.08 & 0.33 & 12.8 \\
\hline SB & 26 & SM & 20 & 40 & 9840 & 8880 & 0.10 & 0.90 & 73 & 0.27 & 44765 & 2.4 & 0.998 & -0.34 & -2.58 & 10.2 \\
\hline SW & 10 & SM & 20 & 40 & 259 & 200 & 0.34 & 0.66 & 75 & 0.23 & 889 & 6.7 & 0.996 & -3.97 & -1.87 & 26.8 \\
\hline BV2 & 14 & $\mathrm{PE}$ & 25 & 40 & 903 & 800 & 0.20 & 0.80 & 131 & 1.62 & 562 & 1.7 & 0.998 & -0.06 & -0.09 & 21.7 \\
\hline BV2 & 16 & PE & 15 & 40 & 193 & 200 & 0.15 & 0.85 & 93 & 0.16 & 149 & 7.2 & 0.993 & -2.56 & -0.43 & 12.2 \\
\hline BV2 & 18 & SM & 15 & 40 & 193 & 200 & 0.16 & 0.84 & 81 & 0.21 & 211 & 7.5 & 0.994 & -2.83 & -0.53 & 13.5 \\
\hline BV2 & 16 & PE & 15 & 40 & 386 & 400 & 0.16 & 0.84 & 83 & 0.18 & 538 & 6.7 & 0.994 & -2.11 & -0.73 & 12.8 \\
\hline BV2 & 18 & SM & 15 & 40 & 386 & 400 & 0.16 & 0.84 & 69 & 0.15 & 708 & 7.2 & 0.996 & -3.55 & -1.26 & 13.3 \\
\hline BV2 & 13 & PE & 20 & 40 & 226 & 200 & 0.21 & 0.79 & 77 & 0.50 & 67 & 3.5 & 0.998 & -1.24 & -0.35 & 17.7 \\
\hline BV2 & 10 & SM & 20 & 40 & 226 & 200 & 0.16 & 0.84 & 77 & 0.15 & 86 & 4.8 & 0.995 & -0.92 & -0.19 & 12.8 \\
\hline BV2 & 13 & PE & 20 & 40 & 451 & 400 & 0.22 & 0.78 & 83 & 0.26 & 636 & 5.4 & 0.997 & -2.52 & -1.42 & 17.5 \\
\hline BV2 & 10 & SM & 20 & 40 & 451 & 400 & 0.15 & 0.85 & 94 & 0.17 & 945 & 8.3 & 0.995 & -4.58 & -1.81 & 12.5 \\
\hline BV2 & 14 & PE & 20 & 40 & 903 & 800 & 0.18 & 0.82 & 94 & 0.22 & 1231 & 3.7 & 0.995 & -0.45 & -0.46 & 14.8 \\
\hline BV2 & 14 & SM & 20 & 40 & 903 & 800 & 0.16 & 0.84 & 75 & 0.18 & 3783 & 7.8 & 0.995 & -4.71 & -4.04 & 13.4 \\
\hline BV2 & 14 & $P E$ & 25 & 40 & 226 & 200 & 0.19 & 0.81 & 105 & 0.35 & 24 & 1.8 & 0.998 & -0.09 & -0.03 & 16.2 \\
\hline BV2 & 14 & SM & 25 & 40 & 226 & 200 & 0.15 & 0.85 & 104 & 0.30 & 64 & 3.5 & 0.997 & -1.05 & -0.26 & 13.7 \\
\hline BV2 & 14 & PE & 25 & 40 & 451 & 400 & 0.19 & 0.81 & 95 & 0.22 & 129 & 2.1 & 0.998 & -0.22 & -0.13 & 16.3 \\
\hline BV2 & 14 & SM & 25 & 40 & 451 & 400 & 0.16 & 0.84 & 98 & 0.20 & 681 & 5.5 & 0.995 & -2.23 & -1.16 & 14.3 \\
\hline BV2 & 14 & SM & 25 & 40 & 903 & 800 & 0.20 & 0.80 & 81 & 0.18 & 1779 & 3.7 & 0.998 & -1.70 & -2.08 & 17.3 \\
\hline
\end{tabular}

896 EOM: exogenous organic matter; WHC: water holding capacity; DEOM: decomposable EOM; REOM: resistant

897 EOM; HEOM: humified EOM; f: partitioning coefficient; K: decomposition constant rate $\left(\mathrm{y}^{-1}\right)$; SSR: sum of

898 squared residuals; RMSE: root mean square error; R: coefficient of correlation; E: relative error; M: mean

899 difference; $\mathrm{CO}_{2}-\mathrm{C}$ : cumulative net $\mathrm{CO}_{2}-\mathrm{C}$ emission in amended soil.

900 For EOM code refer to Table 3, for soil code refer to Table 2. 
Table S4c. Incubation conditions, exogenous organic matter (EOM) pool parameters from model optimization, statistical indicators of model goodness of fit and cumulative net respiration for meat and bone meals.

\begin{tabular}{|c|c|c|c|c|c|c|c|c|c|c|c|c|c|c|c|c|}
\hline EOM & Days & Soil & $\begin{array}{c}\text { Temp } \\
{ }^{\circ} \mathrm{C}\end{array}$ & WHC & $\begin{array}{l}\text { EOM rate } \\
\mu \mathrm{gCg}{ }^{-1}\end{array}$ & $\begin{array}{l}\text { EOM rate } \\
\mathrm{kg} \mathrm{N} \mathrm{ha}^{-1}\end{array}$ & $f_{D E O M}$ & $\mathbf{f}_{\text {REOM }}$ & $K_{\text {DEOM }}$ & $K_{\text {REOM }}$ & $\begin{array}{c}\text { SSR } \\
\mu \mathrm{gCg} \mathrm{g}^{-1}\end{array}$ & $\begin{array}{c}\text { RMSE } \\
\%\end{array}$ & $\mathbf{R}$ & $\begin{array}{l}\mathbf{E} \\
\%\end{array}$ & $\begin{array}{c}M \\
\mu g C g^{-1}\end{array}$ & $\begin{array}{c}\mathrm{CO}_{2}-\mathrm{C} \\
\%\end{array}$ \\
\hline BV2 & 13 & $P E$ & 20 & 40 & 87 & 100 & 0.17 & 0.83 & 85 & 1.66 & 3 & 1.7 & 0.999 & 0.02 & 0.00 & 18.2 \\
\hline BV2 & 14 & SM & 20 & 40 & 87 & 100 & 0.17 & 0.8301 & 73 & 0.39 & 7 & 2.8 & 0.998 & -0.42 & -0.04 & 15.3 \\
\hline BV2 & 14 & SM & 20 & 40 & 175 & 200 & 0.18 & 0.82 & 83 & 0.43 & 38 & 3.0 & 0.998 & -0.73 & -0.16 & 16.5 \\
\hline BV2 & 14 & SM & 20 & 40 & 349 & 400 & 0.17 & 0.83 & 88 & 0.63 & 253 & 3.9 & 0.998 & -1.50 & -0.63 & 16.5 \\
\hline BV2 & 14 & SM & 20 & 40 & 524 & 600 & 0.20 & 0.80 & 68 & 0.16 & 481 & 3.5 & 0.999 & -1.46 & -0.93 & 17.1 \\
\hline BV2 & 13 & PE & 20 & 40 & 175 & 200 & 0.17 & 0.83 & 91 & 1.00 & 7 & 1.4 & 0.999 & 0.00 & 0.00 & 16.1 \\
\hline BV2 & 13 & PE & 20 & 40 & 349 & 400 & 0.19 & 0.81 & 73 & 0.46 & 45 & 1.8 & 0.999 & -0.21 & -0.08 & 16.0 \\
\hline BV2 & 13 & PE & 20 & 40 & 524 & 600 & 0.19 & 0.81 & 73 & 0.31 & 85 & 1.7 & 0.999 & -0.18 & -0.10 & 15.6 \\
\hline BV2 & 9 & SM & 20 & 40 & 211 & 200 & 0.14 & 0.86 & 58 & 1.40 & 12 & 2.6 & 0.999 & -0.23 & -0.04 & 13.7 \\
\hline BV2 & 14 & PE & 25 & 40 & 844 & 800 & 0.15 & 0.85 & 121 & 1.36 & 210 & 1.5 & 0.999 & 0.03 & 0.04 & 17.0 \\
\hline BV2 & 16 & PE & 15 & 40 & 216 & 200 & 0.10 & 0.90 & 64 & 0.19 & 27 & 4.6 & 0.996 & -1.11 & -0.12 & 7.9 \\
\hline BV2 & 18 & SM & 15 & 40 & 216 & 200 & 0.10 & 0.90 & 74 & 0.20 & 56 & 5.6 & 0.996 & -2.32 & -0.30 & 8.5 \\
\hline BV2 & 16 & PE & 15 & 40 & 431 & 400 & 0.10 & 0.90 & 62 & 0.22 & 125 & 5.0 & 0.997 & -1.65 & -0.37 & 7.8 \\
\hline BV2 & 18 & SM & 15 & 40 & 431 & 400 & 0.12 & 0.88 & 62 & 0.16 & 268 & 5.8 & 0.997 & -3.05 & -0.83 & 9.4 \\
\hline BV2 & 13 & PE & 20 & 40 & 211 & 200 & 0.17 & 0.83 & 71 & 0.50 & 35 & 3.4 & 0.997 & -1.08 & -0.23 & 14.7 \\
\hline BV2 & 13 & PE & 20 & 40 & 422 & 400 & 0.15 & 0.85 & 62 & 0.17 & 58 & 2.7 & 0.999 & -0.83 & -0.29 & 11.8 \\
\hline BV2 & 9 & SM & 20 & 40 & 422 & 400 & 0.16 & 0.84 & 57 & 0.20 & 55 & 3.2 & 0.999 & -1.22 & -0.37 & 11.5 \\
\hline BV2 & 14 & PE & 20 & 40 & 844 & 800 & 0.12 & 0.88 & 97 & 0.61 & 213 & 2.3 & 0.998 & -0.20 & -0.14 & 11.1 \\
\hline BV2 & 14 & SM & 20 & 40 & 844 & 800 & 0.12 & 0.88 & 64 & 0.16 & 522 & 4.2 & 0.997 & -1.61 & -0.94 & 9.9 \\
\hline BV2 & 14 & PE & 25 & 40 & 211 & 200 & 0.14 & 0.86 & 96 & 0.35 & 7 & 1.4 & 0.999 & 0.04 & 0.01 & 12.5 \\
\hline BV2 & 14 & SM & 25 & 40 & 211 & 200 & 0.13 & 0.87 & 84 & 0.18 & 11 & 1.9 & 0.998 & -0.17 & -0.03 & 11.5 \\
\hline BV2 & 14 & PE & 25 & 40 & 422 & 400 & 0.14 & 0.86 & 96 & 0.38 & 22 & 1.2 & 0.999 & 0.00 & 0.00 & 12.4 \\
\hline BV2 & 14 & SM & 25 & 40 & 422 & 400 & 0.12 & 0.88 & 99 & 0.19 & 174 & 4.0 & 0.997 & -1.65 & -0.60 & 10.7 \\
\hline BV2 & 14 & SM & 25 & 40 & 844 & 800 & 0.16 & 0.84 & 74 & 0.19 & 1972 & 5.4 & 0.995 & -2.87 & -2.57 & 14.0 \\
\hline $\mathrm{DE}$ & 9 & GO & 20 & 40 & 187 & 200 & 0.23 & 0.77 & 76 & 1.34 & 52 & 3.9 & 0.998 & -0.96 & -0.23 & 19.5 \\
\hline $\mathrm{DE}$ & 9 & $\mathrm{BU}$ & 20 & 40 & 187 & 200 & 0.28 & 0.72 & 31 & 0.40 & 108 & 9.4 & 0.993 & -4.89 & -0.70 & 13.7 \\
\hline $\mathrm{DE}$ & 12 & $\mathrm{BU}$ & 20 & 40 & 374 & 400 & 0.18 & 0.82 & 42 & 0.33 & 638 & 11 & 0.992 & -5.63 & -1.49 & 11.7 \\
\hline $\mathrm{DE}$ & 9 & GO & 20 & 40 & 93 & 100 & 0.31 & 0.69 & 87 & 0.28 & 55 & 6.6 & 0.996 & -3.57 & -0.53 & 23.3 \\
\hline $\mathrm{DE}$ & 9 & SM & 20 & 40 & 93 & 100 & 0.44 & 0.56 & 48 & 0.76 & 13 & 2.7 & 0.999 & -0.25 & -0.04 & 30.9 \\
\hline $\mathrm{DE}$ & 9 & SM & 20 & 40 & 187 & 200 & 0.38 & 0.62 & 52 & 0.15 & 62 & 3.4 & 0.998 & -0.58 & -0.18 & 26.0 \\
\hline $\mathrm{DE}$ & 9 & LO & 20 & 40 & 187 & 200 & 0.50 & 0.50 & 56 & 0.15 & 70 & 2.9 & 0.998 & -0.45 & -0.17 & 32.6 \\
\hline $\mathrm{DE}$ & 9 & SM & 20 & 40 & 374 & 400 & 0.32 & 0.68 & 60 & 0.17 & 494 & 5.4 & 0.998 & -3.36 & -1.82 & 23.1 \\
\hline $\mathrm{DE}$ & 9 & GO & 20 & 40 & 374 & 400 & 0.26 & 0.74 & 62 & 0.15 & 201 & 4.2 & 0.996 & -0.64 & -0.28 & 18.0 \\
\hline $\mathrm{DE}$ & 12 & LO & 20 & 40 & 374 & 400 & 0.34 & 0.66 & 71 & 0.15 & 595 & 4.0 & 0.998 & -1.34 & -0.93 & 26.2 \\
\hline mean & & & & & & & 0.20 & 0.80 & 76 & 0.41 & 2133 & 4.6 & 0.997 & -1.73 & -0.71 & 16.1 \\
\hline minimum & & & & & & & 0.07 & 0.37 & 13 & 0.15 & 3 & 1.2 & 0.989 & -11.18 & -5.52 & 7.8 \\
\hline maximum & & & & & & & 0.63 & 0.93 & 131 & 1.66 & 44765 & 16.1 & 0.999 & 0.75 & 2.11 & 38.6 \\
\hline
\end{tabular}

906 EOM: exogenous organic matter; WHC: water holding capacity; DEOM: decomposable EOM; REOM: resistant

907 EOM; HEOM: humified EOM; f: partitioning coefficient; K: decomposition constant rate ( $\left.\mathrm{y}^{-1}\right)$; SSR: sum of

908 squared residuals; RMSE: root mean square error; R: coefficient of correlation; E: relative error; M: mean 909 difference; $\mathrm{CO}_{2}-\mathrm{C}$ : cumulative net $\mathrm{CO}_{2}-\mathrm{C}$ emission in amended soil.

910 For EOM code refer to Table 3, for soil code refer to Table 2. 
Table S5. Incubation conditions, exogenous organic matter (EOM) pool parameters from model optimization, statistical indicators of model goodness of fit and cumulative net respiration for animal residues.

\begin{tabular}{|c|c|c|c|c|c|c|c|c|c|c|c|c|c|c|c|c|}
\hline EOM & Days & Soil & $\begin{array}{c}\text { Temp } \\
{ }^{\circ} \mathrm{C}\end{array}$ & WHC & $\begin{array}{c}\text { EOM rate } \\
\%\end{array}$ & $\begin{array}{l}\text { C rate } \\
\mu g g^{-1}\end{array}$ & $f_{D E O M}$ & $\mathbf{f}_{\text {REOM }}$ & $\mathrm{K}_{\mathrm{DEOM}}$ & $\mathrm{K}_{\text {REOM }}$ & $\begin{array}{c}\text { SSR } \\
\mu g \mathrm{Cg}^{-1}\end{array}$ & $\begin{array}{c}\text { RMSE } \\
\%\end{array}$ & $\mathbf{R}$ & $\begin{array}{l}\mathbf{E} \\
\%\end{array}$ & $\begin{array}{c}M \\
\mu g \mathrm{Cg}^{-1}\end{array}$ & $\begin{array}{c}\mathrm{CO}_{2}-\mathrm{C} \\
\% \\
\end{array}$ \\
\hline $\mathrm{HL}$ & 27 & JU & 20 & 40 & 0.5 & 2288 & 0.12 & 0.88 & 92 & 1.09 & 27066 & 5.4 & 0.997 & -3.88 & -9.59 & 15.8 \\
\hline $\mathrm{HL}$ & 27 & LO & 20 & 40 & 0.5 & 2288 & & 0.79 & 82 & 0.35 & 14820 & 2.5 & 0.997 & -0.30 & -1.08 & 19.5 \\
\hline $\mathrm{HL}$ & 25 & SM & 20 & 40 & 0.5 & 2288 & 0.13 & 0.87 & 28 & 0.58 & 8953 & 3.7 & 0.997 & -0.05 & -0.09 & 13.5 \\
\hline BLM & 26 & SM & 20 & 40 & 0.5 & 2874 & 0.04 & 0.96 & 132 & 1.20 & 35527 & 7.9 & 0.983 & -1.33 & -2.72 & 12.2 \\
\hline BLM & 27 & JU & 20 & 40 & 0.5 & 2874 & 0.06 & 0.94 & 144 & 0.87 & 38469 & 7.3 & 0.980 & 1.34 & 2.90 & 11.0 \\
\hline BLM & 7 & JU & 20 & 40 & 0.5 & 2874 & 0.15 & 0.85 & 111 & 0.15 & 16407 & 9.4 & 0.991 & -3.18 & -6.55 & 10.7 \\
\hline BLM & 27 & LO & 20 & 40 & 0.5 & 2874 & 0.22 & 0.78 & 110 & 0.45 & 75279 & 4.3 & 0.989 & -0.43 & -2.12 & 20.4 \\
\hline BLM & 26 & SM & 20 & 40 & 1.0 & 5749 & 0.07 & 0.93 & 99 & 0.16 & 57885 & 6.3 & 0.983 & -0.85 & -2.80 & 7.2 \\
\hline BLM & 26 & SM & 20 & 40 & 2.0 & 11497 & 0.05 & 0.95 & 131 & 0.15 & 99517 & 5.9 & 0.981 & -0.81 & -3.67 & 5.0 \\
\hline BLM & 35 & JU & 10 & 20 & 0.5 & 2631 & 0.07 & 0.93 & 189 & 0.57 & 12399 & 5.4 & 0.993 & -1.16 & -1.58 & 6.8 \\
\hline BLM & 35 & JU & 10 & 30 & 0.5 & 2631 & 0.07 & 0.93 & 139 & 0.15 & 11499 & 5.8 & 0.987 & -0.15 & -0.18 & 5.8 \\
\hline BLM & 35 & JU & 10 & 40 & 0.5 & 2631 & 0.09 & 0.91 & 111 & 0.15 & 21366 & 6.3 & 0.985 & 0.68 & 1.04 & 7.2 \\
\hline BLM & 36 & JU & 20 & 20 & 0.5 & 2631 & 0.10 & 0.90 & 185 & 0.63 & 4693 & 1.9 & 0.998 & -0.18 & -0.42 & 11.2 \\
\hline BLM & 36 & JU & 20 & 30 & 0.5 & 2631 & 0.09 & 0.91 & 189 & 0.60 & 5951 & 2.2 & 0.996 & -0.15 & -0.35 & 11.4 \\
\hline BLM & 36 & JU & 20 & 40 & 0.5 & 2631 & 0.10 & 0.90 & 155 & 0.80 & 11305 & 2.4 & 0.996 & -0.15 & -0.44 & 14.9 \\
\hline BLM & 35 & JU & 30 & 20 & 0.5 & 2631 & 0.12 & 0.88 & 261 & 0.37 & 4466 & 1.8 & 0.996 & 0.55 & 1.51 & 12.6 \\
\hline BLM & 35 & JU & 30 & 30 & 0.5 & 2631 & 0.12 & 0.88 & 259 & 0.24 & 3266 & 1.6 & 0.996 & -0.05 & -0.14 & 12.1 \\
\hline BLM & 35 & JU & 30 & 40 & 0.5 & 2631 & 0.13 & 0.87 & 208 & 0.34 & 4569 & 1.6 & 0.997 & -0.05 & -0.15 & 15.1 \\
\hline BLM & 27 & JU & 20 & 40 & 0.5 & 2692 & 0.15 & 0.85 & 119 & 0.69 & 17033 & 2.9 & 0.995 & -0.32 & -1.07 & 15.8 \\
\hline BLM & 27 & LO & 20 & 40 & 0.5 & 2692 & 0.19 & 0.81 & 247 & 0.82 & 13951 & 2.0 & 0.995 & -0.10 & -0.43 & 19.7 \\
\hline BLM & 25 & SM & 20 & 40 & 0.5 & 2692 & 0.07 & 0.93 & 284 & 1.21 & 16804 & 3.9 & 0.992 & -0.21 & -0.53 & 13.9 \\
\hline HHM & 27 & JU & 20 & 40 & 0.5 & 2975 & 0.40 & 0.60 & 11 & 0.15 & 116849 & 8.6 & 0.998 & -6.16 & -19.78 & 20.3 \\
\hline HHM & 27 & LO & 20 & 40 & 0.5 & 2975 & 0.28 & 0.72 & 25 & 0.15 & 126675 & 6.3 & 0.994 & -0.90 & -3.81 & 21.1 \\
\hline HHM & 25 & SM & 20 & 40 & 0.5 & 2975 & 0.33 & 0.67 & 14 & 0.15 & 728880 & 20.2 & 0.987 & -13.64 & -44.80 & 20.6 \\
\hline HHM & 35 & JU & 10 & 20 & 0.5 & 2565 & 0.04 & 0.96 & 12 & 2.51 & 56817 & 21.8 & 0.988 & -16.44 & -11.77 & 6.3 \\
\hline HHM & 35 & JU & 10 & 30 & 0.5 & 2565 & 0.08 & 0.92 & 38 & 0.55 & 53050 & 17.9 & 0.990 & -13.34 & -11.26 & 6.3 \\
\hline HHM & 35 & JU & 10 & 40 & 0.5 & 2565 & 0.14 & 0.86 & 17 & 0.28 & 10226 & 7.0 & 0.998 & -3.71 & -3.53 & 6.8 \\
\hline HHM & 36 & JU & 20 & 20 & 0.5 & 2565 & 0.24 & 0.76 & 15 & 0.15 & 82303 & 9.3 & 0.996 & -5.36 & -10.73 & 13.8 \\
\hline HHM & 36 & JU & 20 & 30 & 0.5 & 2565 & 0.22 & 0.78 & 15 & 0.15 & 61902 & 7.3 & 0.997 & -3.62 & -8.05 & 14.4 \\
\hline HHM & 36 & JU & 20 & 40 & 0.5 & 2565 & 0.24 & 0.76 & 14 & 0.15 & 79363 & 7.0 & 0.998 & -4.64 & -12.00 & 16.6 \\
\hline HHM & 35 & JU & 30 & 20 & 0.5 & 2565 & 0.13 & 0.87 & 13 & 0.29 & 19209 & 7.1 & 0.998 & -4.35 & -6.25 & 10.9 \\
\hline $\mathrm{HHM}$ & 35 & JU & 30 & 30 & 0.5 & 2565 & 0.16 & 0.84 & 23 & 0.23 & 79358 & 8.8 & 0.993 & -3.86 & -9.11 & 14.6 \\
\hline $\mathrm{HHM}$ & 35 & JU & 30 & 40 & 0.5 & 2565 & 0.19 & 0.81 & 18 & 0.20 & 58410 & 6.2 & 0.996 & -2.71 & -7.73 & 17.3 \\
\hline mean & & & & & & & 0.15 & 0.85 & 106 & 0.50 & 59826 & 6.6 & 0.993 & -2.71 & -5.37 & 13.1 \\
\hline minimum & & & & & & & 0.04 & 0.60 & 11 & 0.15 & 3266 & 1.6 & 0.980 & -16.44 & -44.80 & 5.0 \\
\hline maximum & & & & & & & 0.40 & 0.96 & 284 & 2.51 & 728880 & 21.8 & 0.998 & 1.34 & 2.90 & 21.1 \\
\hline
\end{tabular}

EOM: exogenous organic matter; WHC: water holding capacity; DEOM: decomposable EOM; REOM: resistant

916 EOM; HEOM: humified EOM; f: partitioning coefficient; K: decomposition constant rate $\left(\mathrm{y}^{-1}\right)$; SSR: sum of 917 squared residuals; RMSE: root mean square error; R: coefficient of correlation; E: relative error; M: mean 918 difference; $\mathrm{CO}_{2}-\mathrm{C}$ : cumulative net $\mathrm{CO}_{2}-\mathrm{C}$ emission in amended soil. For EOM code refer to Table 3, for soil code refer to Table 2 . 
921 Table S6. Incubation conditions, exogenous organic matter (EOM) pool parameters from model optimization, statistical indicators of model goodness of fit and cumulative net respiration for crop residues, agro-industrial wastes and sewage sludges.

\begin{tabular}{|c|c|c|c|c|c|c|c|c|c|c|c|c|c|c|c|c|c|}
\hline EOM & Days & Soil & $\begin{array}{c}\text { Temp } \\
{ }^{\circ} \mathrm{C}\end{array}$ & WHC & $\begin{array}{c}\text { EOM } \\
\text { rate } \\
\% \\
\end{array}$ & $\begin{array}{l}\text { Crate } \\
\mu g^{-1}\end{array}$ & $f_{\text {DEOM }}$ & $f_{\text {REOM }}$ & $\mathbf{f}_{\text {HEOM }}$ & $K_{D E O M}$ & $K_{\text {REOM }}$ & $\begin{array}{c}\text { SSR } \\
\mu \mathrm{gC} \mathrm{g}^{-1}\end{array}$ & $\begin{array}{c}\text { RMSE } \\
\%\end{array}$ & $\mathbf{R}$ & $\begin{array}{l}\mathbf{E} \\
\%\end{array}$ & $\begin{array}{c}\mathrm{M} \\
\mu \mathrm{gC} g^{-1}\end{array}$ & $\begin{array}{c}\mathrm{CO}_{2}-\mathrm{C} \\
\%\end{array}$ \\
\hline \multicolumn{18}{|c|}{ Crop residues (CR) } \\
\hline $\mathrm{CC}$ & 26.7 & LO & 20 & 40 & 0.5 & 2529 & 0.09 & 0.91 & & 75 & 1.53 & 2433 & 1.3 & 0.9996 & 0.21 & 0.62 & 17.4 \\
\hline CC & 26.8 & JU & 20 & 40 & 0.5 & 2529 & 0.04 & 0.96 & & 94 & 0.65 & 535 & 1.6 & 0.9993 & 0.21 & 0.24 & 7.2 \\
\hline CC & 25.9 & SM & 20 & 40 & 0.5 & 2529 & 0.08 & 0.92 & & 32 & 0.16 & 9406 & 6.0 & 0.9927 & -3.23 & -4.14 & 7.4 \\
\hline CC & 25.9 & SM & 20 & 40 & 1.0 & 5058 & 0.04 & 0.96 & & 72 & 0.31 & 6851 & 3.1 & 0.9959 & -0.17 & -0.35 & 5.7 \\
\hline CC & 25.9 & SM & 20 & 40 & 2.0 & 10116 & 0.03 & 0.97 & & 148 & 0.29 & 5785 & 1.8 & 0.9982 & 0.19 & 0.65 & 4.6 \\
\hline WS & 26.7 & LO & 20 & 40 & 0.5 & 2748 & 0.17 & 0.83 & & 26 & 0.94 & 8610 & 2.2 & 0.9990 & -0.34 & -1.06 & 18.4 \\
\hline WS & 26.8 & JU & 20 & 40 & 0.5 & 2748 & 0.04 & 0.96 & & 48 & 0.30 & 464 & 1.9 & 0.9991 & -0.22 & -0.20 & 5.1 \\
\hline WS & 25.9 & SM & 20 & 40 & 0.5 & 2748 & 0.08 & 0.92 & & 37 & 0.17 & 46962 & 12.9 & 0.9878 & -6.62 & -8.79 & 7.2 \\
\hline WS & 25.9 & SM & 20 & 40 & 1.0 & 5495 & 0.05 & 0.95 & & 28 & 0.15 & 14186 & 5.2 & 0.9944 & -0.87 & -1.56 & 4.8 \\
\hline WS & 25.9 & SM & 20 & 40 & 2.0 & 10991 & 0.02 & 0.98 & & 42 & 0.15 & 2561 & 1.8 & 0.9990 & -0.02 & -0.04 & 3.0 \\
\hline \multicolumn{18}{|c|}{ Agro-industrial wastes (AW) } \\
\hline TPOMW & 26.7 & LO & 20 & 40 & 0.5 & 2796 & 0.06 & 0.75 & 0.19 & 230 & 2.40 & 1593 & 1.2 & 0.9995 & -0.23 & -0.60 & 17.5 \\
\hline TPOMW & 26.5 & JU & 20 & 40 & 0.5 & 2796 & 0.03 & 0.79 & 0.19 & 119 & 0.81 & 845 & 1.8 & 0.9990 & 0.10 & 0.12 & 6.6 \\
\hline TPOMW & 24.8 & SM & 20 & 40 & 0.5 & 2796 & 0.05 & 0.76 & 0.19 & 132 & 0.31 & 2978 & 3.3 & 0.9952 & -1.60 & -2.07 & 6.0 \\
\hline \multicolumn{18}{|c|}{ Sewage sludges (SS) } \\
\hline WW & 28.8 & SM & 20 & 40 & 0.5 & 1920 & 0.04 & 0.96 & & 45 & 0.31 & 177 & 1.2 & 0.9995 & 0.13 & 0.10 & 6.0 \\
\hline WW & 28.8 & $\mathrm{PE}$ & 20 & 40 & 0.5 & 1920 & 0.04 & 0.96 & & 83 & 0.26 & 203 & 1.5 & 0.9989 & 0.09 & 0.06 & 4.9 \\
\hline WW & 28.8 & LL & 20 & 40 & 0.5 & 1920 & 0.03 & 0.97 & & 62 & 0.15 & 260 & 2.0 & 0.9981 & -0.10 & -0.06 & 3.8 \\
\hline WW & 28.8 & $A L$ & 20 & 40 & 0.5 & 1920 & 0.04 & 0.96 & & 62 & 0.15 & 1181 & 4.4 & 0.9975 & 3.49 & 2.19 & 4.5 \\
\hline \multirow[t]{3}{*}{ CR } & mean & & & & & & 0.06 & 0.94 & & 60 & 0.47 & 9779 & 3.8 & 0.9965 & -1.08 & -1.32 & 8.5 \\
\hline & minimum & & & & & & 0.02 & 0.83 & & 26 & 0.15 & 464 & 1.3 & 0.9878 & -6.62 & -8.79 & 3.0 \\
\hline & maximum & & & & & & 0.17 & 0.98 & & 148 & 1.53 & 46962 & 12.9 & 0.9996 & 0.21 & 0.65 & 18.4 \\
\hline \multirow[t]{3}{*}{ AW } & mean & & & & & & 0.05 & 0.77 & 0.19 & 160 & 1.17 & 1805 & 2.1 & 0.9979 & -0.57 & -0.85 & 10.0 \\
\hline & minimum & & & & & & 0.03 & 0.75 & 0.19 & 119 & 0.31 & 845 & 1.2 & 0.9952 & -1.60 & -2.07 & 6.0 \\
\hline & maximum & & & & & & 0.06 & 0.79 & 0.19 & 230 & 2.40 & 2978 & 3.3 & 0.9995 & 0.10 & 0.12 & 17.5 \\
\hline \multirow[t]{3}{*}{ SS } & mean & & & & & & 0.04 & 0.96 & & 63 & 0.22 & 455 & 2.3 & 0.9985 & 0.90 & 0.57 & 4.8 \\
\hline & minimum & & & & & & 0.03 & 0.96 & & 45 & 0.15 & 177 & 1.2 & 0.9975 & -0.10 & -0.06 & 3.8 \\
\hline & maximum & & & & & & 0.04 & 0.97 & & 83 & 0.31 & 1181 & 4.4 & 0.9995 & 3.49 & 2.19 & 6.0 \\
\hline
\end{tabular}

EOM: exogenous organic matter; WHC: water holding capacity; DEOM: decomposable EOM; REOM: resistant

926 EOM; HEOM: humified EOM; f: partitioning coefficient; K: decomposition constant rate $\left(\mathrm{y}^{-1}\right)$; SSR: sum of

927 squared residuals; RMSE: root mean square error; R: coefficient of correlation; E: relative error; M: mean

928 difference; $\mathrm{CO}_{2}-\mathrm{C}$ : cumulative net $\mathrm{CO}_{2}-\mathrm{C}$ emission in amended soil.

929 For EOM code refer to Table 3, for soil code refer to Table 2. 